\title{
Unsteady Collisionless Gaseous Plasma Flow in the Upper Layers of the Charged Atmosphere and The Investigation of Vlasov-Maxwell Equations
} System.

Taha Zakaraia Abdel Wahid ${ }^{1, *}$, Member, IEEE Adel, M. Morad ${ }^{1,2}$, Mohamad Almakrami ${ }^{3}$, Tarik Lamoudan ${ }^{4}$,

${ }^{1}$ Mathematics and Computer Science Department, Faculty of Science, Menofia University, Shebin El-Kom 32511, Egypt.

${ }^{2}$ Department of Computational Mathematics and Mathematical Physics, Institute of Mathematics, Mechanics and Computer Science, Southern Federal University, Rostov on Don 344090, Russia.

${ }^{3}$ Faculty of Education, Al Hodeidah University, Al-Hodeidah, Yemen.

${ }^{4}$ Department of Mathematics, Faculty of Science and Arts, King Khalid University, Mahail City, Kingdom of Saudi Arabia.

* Corresponding author Emails: taha_zakaraia@yahoo.com;

This work is supported by the Egyptian Academy of Scientific Research and Technology by the associated grant number (No.6508), under the program of ScienceUP Faculties of Science.

\section{Abstract}

In the upper charged layers of the atmosphere, the plasma is very rarefied. The collisions between its molecules are almost non-existent, and the driving forces behind them are the Lorentz forces resulting from the electric and magnetic fields. For this reason, we are interested in studying the behavior of non-collision plasmas because of its essential applications, such as the movement of satellites in the charged atmosphere. In this paper, the flow problem of collisionless gaseous plasma is examined. For that propose, we solve the unsteady Vlasov-Maxwell system of non-linear partial differential equations analytically. Methods of moments and traveling wave parameters are used to acquire an exact solution. Specific macroscopic properties of collisionless gaseous plasma are calculated along with electrical and magnetic fields. Further, thermodynamic estimation, such as entropy and entropy production, is presented. Those calculations allow us to measure the consistency with the laws of non-equilibrium thermodynamics. Relations between internal energy modification participations are predicted using Gibbs' equation for collisionless plasma. The modification effect of internal energies due to electro-magnetic fields is found to be small compared with the internal energy change due to the effect of entropy. That is because these fields are self-induced by plasma particles due to the sudden movement of the rigid plane plate. The results are accomplished according to the typical argon gaseous plasma model. Three-dimensional diagrams showing the measured variables are drawn to investigate and discuss their behavior. The problem has many commercial applications for the movement of objects in the charged atmosphere.

INDEX TERMS Collisionless plasma dynamics; Gaseous plasma; Vlasov equation; Maxwell equations; Entropy; Extended Gibbs formula; The Earth's plasmasphere; the magnetosphere.

\section{PACS: 52.27.Aj; 52.25.Kn; 51.10.+y; 52.30.-q; 71.10.Ca; 79.20.Uv.}

\section{Introduction}

The manner of highly rarefied gaseous plasma near an illimitable flat rigid plate suddenly moved in its plane is of great interest in aerodynamic and many various vital applications in engineering and industry. The flow over an endless moving plane may be considered as a critical problem in gas dynamics [1-10], since Stokes (1901), Lord Rayleigh (1911), discussed the flow about an illimitable plane wall that executes linear harmonic oscillation parallel to itself.

Also, Cericignani and Sernagiotto (1965) have used the kinetic theory to investigate the manner of a rarefied gas over an illimitable plane that suddenly moved in its plane. Sommerfeld (1967) investigated the flow of slightly rarefied gas over an illimitable plane wall based on a relaxation model of Boltzmann's equation. Yoshio and Kyoii (1971) studied the gas flow over an illimitable plane wall because of the relaxation model of Boltzmann's equation. Broadwell (1976) and Gatignol (1977), who analyzed single-component gas using discrete velocity models, achieved results in a qualitative agreement with the results obtained by the linearized BGK framework of Boltzmann's equation. Ramaand Agarwal (1978) used a finite difference method to solve the unsteady flow past a flat rigid plate numerically. The unsteady viscous fluid stress on a flat rigid plate was computed by Rolf (1980) using a simplified unsteady momentum equation that was not restricted by a frequency parameter. He compared favorably with the available calculations. The Rayleigh flow problem of gases in a highly rarefied environment, which is formed by a homogeneous system of plasma particles, has been studied by Khater and El-Sharif [11] within the framework of the theory of gas kinetics. They examined the dynamical and electro-magnetic manner of the gaseous plasma. Besides, they solved the problem approximately using the perturbation techniques, and therefore, their solutions were discontinuous due to the use of Laplace transformations.

This paper aims to determine the physical macroscopic parameters such as the coefficient of viscosity, velocity, shear stress, coupled with the induced electrical and magnetic fields. We do this by solving the initial boundary value problem of the rarefied gas flow in the collisionless plasma. Moreover, the irreversible thermodynamic properties, which are of fundamental physical importance, are demonstrated using the calculated distribution functions. For achieving this manner, the 
related thermodynamic functions and their physical predictions are investigated.

It is known that thermodynamics of nonequilibrium environment as a kinetic theory describes two closely related approaches to the explanation of nonequilibrium processes in real physical media. Moreover, the direct calculation of the kinetic coefficients is the province of kinetic theory, based on an appropriate model of interactions between the medium particles [12]. It can only establish the general structure of motion equations describing non-equilibrium medium, as well as between the kinetic coefficients in those equations. The role of kinetic theory, however, does not end there. The provision of mathematical apparatus for calculating the transport and relaxation coefficients produced by the kinetic theory, for example, for rarefied gaseous plasma, allows one to determine the applicability of non-equilibrium thermodynamic methods to arbitrary physical media.

In the present study, the irreversible thermodynamic manner of the system is investigated to acquire the entropy, entropy production, and the modification in internal energy. It is shown that the model consists of Onsager's relation with the non-equilibrium thermodynamic properties of the system. Moreover, the distribution function is assumed to satisfy the VlasovBoltzmann equation, and the molecules are reflected from the surface with complete energy accommodation. In the governing equations, the collision term is simplified using the BGK kinetic model proposed by Bhatnagar et al. [13].

Relevant applications within rarefied collisionless gaseous plasma include simulation of the upper atmospheric [14], the Magellan Spacecraft [15], the Space Shuttle Orbiter [16], the Stardust Sample Return Capsule [17], and the Mars Pathfinder [18]. New significance beyond that for research in the upper atmosphere requires chemical vapor deposition [19], the microfilter [20], MEMS, and NEMS [21-24]. The Vlasov equation, based on kinetic theory, has historically remained the only acceptable choice for solving the rarefied collisionless gaseous plasma problems. Also, the Vlasov equation has various applications in MEMS and NEMS technology [25-26].

The aims of this study are: (i) to acquire an exact analytical solution of the unsteady Vlasov's equation for rarefied collisionless gaseous plasma filled in the upper half of the space. The collisionless plasma is bounded by an illimitable flat rigid plate that suddenly moves on its plane. It is producing a self-generated electrical and magnetic field. (ii) to predict the behaviors of the non-equilibrium thermodynamic manner of the introduced model.

\section{The Physical Problem and Mathematical Formulation}

Assuming the upper half-space at $y \geq 0$ is bounded by an infinite, rigid flat plate at $y=0$. It is filled with a collisionless plasma of electrons and positive ions. $\frac{m_{e}}{m_{i}}<<1$ is the mass ratio of electrons to ions in plasma that considered small. The ions will then be considered an immobile neutralizing background. Initially, the collisionless plasma is located in absolute equilibrium, and the wall is at rest. After that, the rigid plate starts to flick along $x$-axis with the velocity, $V_{0} e^{-\omega t}$ with the constants $\omega$ and $V_{0}$. Further, the rigid plate is often known to be insulating, impermeable, and uncharged material. The entire system (electrons + ions + rigid plate) is held at a fixed temperature. The nomenclature specified the physical quantities presented in the context.

The force which acts on the individual electron is given by the Lorentz force [48-51],

$$
\vec{f}_{e}=-e \vec{E}-\frac{e}{C}(\xi \wedge \vec{B})
$$

By assuming,

$$
\left.\begin{array}{cc}
\vec{E} \equiv\left(E_{x}, 0,0\right), & \vec{B} \equiv\left(0,0, B_{z}\right), \\
\vec{V} \equiv\left(V_{x}, 0,0\right), \quad \vec{J} \equiv\left(q n V_{x}, 0,0\right),
\end{array}\right\}
$$

To satisfy Maxwell's equations, let $E_{x}, B_{x}, V_{x}$, and $J_{x}$ are functions of the space component $y$ and time $t$. In collisionless plasma, the distribution function $M_{e}(y, \xi, t)$ of the particles is obtained from the Vlasov equation [4041] as follows,

$$
\frac{\partial M_{e}}{\partial t}+\xi \cdot \frac{\partial M_{e}}{\partial \vec{r}}-\frac{e}{m_{e}}\left(\vec{E}+\frac{1}{C}(\xi \wedge \vec{B})\right) \cdot \frac{\partial M_{e}}{\partial \xi}=0
$$

with

$$
M_{e}=\left\{\begin{array}{l}
M_{1}=n(2 \pi R T)^{-\frac{3}{2}}\left(1+\frac{\xi_{x} V_{x 1}}{R T}\right) \exp \left(\frac{-\xi^{2}}{2 R T}\right) \text { for } \xi_{y}<0 \\
M_{2}=n(2 \pi R T)^{-\frac{3}{2}}\left(1+\frac{\xi_{x} V_{x 2}}{R T}\right) \exp \left(\frac{-\xi^{2}}{2 R T}\right) \text { for } \xi_{y}>0
\end{array},\right.
$$

here $V_{x 1}$ and $V_{x 2}$ are two unknown functions in time, $t$ and the single distance variable, $y$.

The boundary conditions can be formulated, taking into account that the particles are reflected from the rigid plate with a full velocity accommodation. In other words, the collisionless plasma particles are reflected with the rigid plate velocity. Thus the boundary conditions can be written in the form [27],

$$
\begin{aligned}
& V_{x 2}(0, t)=V_{0} e^{-\omega t} \text { for } t>0 \\
& \text { with } V_{x 2}=V_{x} \text { as } \xi_{y}>0 \text { and } V_{x} \text { is finite as } \\
& \mathrm{y} \rightarrow \infty \text {. }
\end{aligned}
$$

Substituting (1) and (2) inside Vlasov's equation (3), one obtains,

$$
\begin{aligned}
& \frac{\partial M_{e}}{\partial t}+\xi_{y} \frac{\partial M_{e}}{\partial y}-\frac{e B_{z}}{m_{e} C}\left(\xi_{y} \frac{\partial M_{e}}{\partial \xi_{x}},\right. \\
& \left.-\xi_{x} \frac{\partial M_{e}}{\partial \xi_{y}}\right)+\frac{e E_{x}}{m_{e}} \frac{\partial M_{e}}{\partial \xi_{x}}=0
\end{aligned}
$$

Solving the Vlasov equation, the moment method of Lees [57-60] is used in our model. For any quantity $Q_{j}(\xi)$

which is a function only of molecular velocity, Maxwell converted Vlasov's equation into an integral transfer equation or moment equation named the Vlasov-Maxwell equation (5). One of the essential advantages of this approach is the unpretentious satisfaction of surface boundary conditions. Multiplying (5) by $Q_{j}(\xi)$ and integrating overall values of $\vec{c}$ following Grad's moment technique [43, 57], the transfer equations will be transformed to the following form 


$$
\begin{aligned}
& \frac{\partial}{\partial t} \int Q_{j} M_{e} d \xi+\frac{\partial}{\partial y} \int \xi_{y} Q_{j} M_{e} d \xi+\frac{e E_{x}}{m_{e}} \int M_{e} \frac{\partial Q_{j}}{\partial \xi_{x}} d \xi \\
& -\frac{e B_{z}}{m_{e} c_{0}} \int\left(\xi_{x} \frac{\partial Q_{j}}{\partial \xi_{y}}-\xi_{y} \frac{\partial Q_{j}}{\partial \xi_{x}}\right) d \xi=0
\end{aligned}
$$

The integrals over the velocities are estimated from the relation [28],

$$
\int Q_{j}(\vec{c}) M d \underline{c}=\int_{-\infty}^{\infty} \int_{-\infty}^{0} \int_{-\infty}^{\infty} Q_{j} M_{1} d \xi+\int_{-\infty}^{\infty} \int_{0}^{\infty} \int_{-\infty}^{\infty} Q_{j} M_{2} d \xi .
$$

Here $\quad Q_{j}=Q_{j}(\vec{c}), j=1,2 \quad$ and $d \xi=d \xi_{x} d \xi_{y} d \xi_{z}$, $\xi_{x}, \xi_{y}$, and $\xi_{z}$ are the particle velocities components along $x-, y-$, and $z$-axes, respectively. Moreover, $E_{x}$ and $B_{z}$ should be obtained from the Maxwell equation,

$\frac{\partial E_{x}}{\partial y}+\frac{1}{C} \frac{\partial B_{z}}{\partial t}=0$

$\frac{\partial B_{z}}{\partial y}-\frac{1}{C} \frac{\partial E_{x}}{\partial t}-\frac{4 \pi e n}{C} V_{x}=0$.

Here $n=\int M d \xi$ and $n V_{x}=\int \xi_{x} M d \xi$ with the boundary and initial conditions,

$$
\left.\begin{array}{l}
E_{x}(y, 0)=B_{z}(y, 0)=0 \\
E_{x}(y, t) \text { and } B_{z}(y, t) \text { are finite as } \mathrm{y} \rightarrow \infty .
\end{array}\right\}
$$

The nondimensionalization process will be applied using the following dimensionless quantities,

$$
\left.\begin{array}{c}
t=t^{\prime} \tau_{e e}, y=y^{\prime}\left(\frac{\tau_{e e} V_{T e}}{\sqrt{2 \pi}}\right), V_{x}=V_{x}^{\prime} V_{T e}, \tau_{x y}=\tau_{x y}^{\prime} V_{T e}, \\
M a=\frac{U_{0}}{V_{T e}}, B_{z}=B_{z}^{\prime}\left(\frac{\sqrt{2 \pi} m_{e} V_{T e}}{e \tau_{e e}}\right), E_{x}^{\prime}=E_{x}^{\prime}\left(\frac{m_{e} V_{T e}}{e \tau_{e e}}\right) \\
, \rho=n m, V_{T e}=\sqrt{\frac{2 k_{B} T_{e}}{m_{e}}}, \varepsilon=\frac{m_{e}}{m_{i}}, d U=d U^{\prime}\left(k_{B} T_{e}\right) \\
\text { and } M_{j}=M_{j} n_{e}\left(2 \pi R T_{e}\right)^{-\frac{3}{2}}, j=0,1,2
\end{array}\right\}
$$

where $\tau_{e e}$ is the relaxation time [ 53-56] and $M a$ is the Mach number ( $M a^{2}<<1$ ), such that the temperature and density alterations are negligible, i.e.

$T=1+O\left(M a^{2}\right), n=1+O\left(M a^{2}\right)$,

Therefore, the velocity and stress tensor can be expressed as follows,

$$
V_{x}=\frac{1}{2}\left(V_{x 1}+V_{x 2}\right), \tau_{x y}=\frac{P_{x y}}{\rho U_{0} \sqrt{R T_{e} / 2 \pi}}=\left(V_{x 2}-V_{x 1}\right) .
$$

Here $P_{x y}$ is the shear stress [31] defined by $P_{x y}=m \int\left(c_{x}-V_{x}\right) c_{y} F d \underline{c}$.

Using the dimensionless transformations in (11), such that $Q_{1}=c_{x}$ and $Q_{2}=c_{x} c_{y}$, and substituting (4) inside (6), after dropping the bars, the following system of equations will be acquired,

$$
\begin{aligned}
& \frac{\partial V_{x}}{\partial t}+\frac{\partial \tau_{x y}}{\partial y}-E_{x}=0, \\
& \frac{\partial \tau_{x y}}{\partial t}+2 \pi \frac{\partial V_{x}}{\partial y}=0, \\
& \frac{\partial E_{x}}{\partial y}+\frac{\partial B_{z}}{\partial t}=0,
\end{aligned}
$$

$$
\frac{\partial B_{z}}{\partial y}-\alpha_{1} \frac{\partial E_{x}}{\partial t}-\alpha_{0} V_{x}=0
$$

Here $\alpha_{0}=\left(\frac{V_{T e}{ }^{2} \tau_{e e}{ }^{2} e^{2} n}{m_{e} C^{2}}\right)$ and $\alpha_{1}=\left(\frac{m_{e} V_{T e}}{\tau_{e e}{ }^{2} e^{2} n C}\right)$. After some algebraic manipulations, taking into account the following initial and boundary conditions,

$$
\begin{gathered}
V_{x}(y, 0)=\tau_{x y}(y, 0)=E_{x}(y, 0)=B_{z}(y, 0)=0, \\
2 V_{x}(0, t)+\tau_{x y}(0, t)=2 M a e^{-\beta_{1} t}, \text { for } t>0 ; \\
V_{x}, \tau_{x y}, E_{x}, \text { and } B_{z} \text { are finite as } \mathrm{y} \rightarrow \infty,
\end{gathered}
$$

The basic system of governing $s$ (14)-(17) will transform to a single differential equation,

$$
\begin{aligned}
& \frac{\partial^{4} V_{x}(y, t)}{\partial t^{2} \partial y^{2}}-2 \pi \frac{\partial^{4} V_{x}(y, t)}{\partial t^{4}}-\alpha_{0} \frac{\partial^{2} V_{x}(y, t)}{\partial t^{2}}= \\
& \alpha_{1} \frac{\partial^{4} V_{x}(y, t)}{\partial t^{4}}-2 \pi \alpha_{1} \frac{\partial^{4} V_{x}(y, t)}{\partial t^{2} \partial y^{2}}=0
\end{aligned} .
$$

\section{Initial-Boundary Value Problem Solution}

In this paper, we employ the traveling wave solution parameter method [59-60] using the traveling wave variable $V_{x}(y, t)=V_{x}(\eta)$ with the transformation,

$$
\eta=l y-m t \text {, }
$$

where $l$ and $m$ are transformation constants to be determined later by the initial and boundary conditions $[59,60]$. In order to get the solution of (19), taking into account (20), to acquire the derivatives,

$$
\frac{\partial}{\partial t}=-m \frac{\partial}{\partial \eta}, \frac{\partial}{\partial y}=l \frac{\partial}{\partial \eta}, \frac{\partial^{n}}{\partial t^{n}}=(-1)^{n} m^{n} \frac{\partial^{n}}{\partial \eta^{n}}, \frac{\partial^{n}}{\partial y^{n}}=l^{n} \frac{\partial^{n}}{\partial \eta^{n}} .
$$

Here $n$ is a positive integer.

Substituting (20) into (19) and using (21) yields the following equation,

$$
\begin{aligned}
& \left(m^{2} l^{2}-2 \pi l^{4}-\alpha_{1} m^{4}+2 \pi \alpha_{1} m^{2} l^{2}\right) \frac{d^{4} V_{x}(\eta)}{d \eta^{4}} \\
& -\alpha_{0} m^{2} \frac{d^{2} V_{x}(\eta)}{d \eta^{2}}=0
\end{aligned}
$$

and the initial and boundary conditions take the form,

$$
\begin{gathered}
E_{x}(\eta=0)=B_{z}(\eta=0)=\tau_{x y}(\eta=0)=0, \\
2 V_{x}(\eta=-m)+\tau_{x y}(\eta=-m)=2 M_{a} e^{-\beta_{1}} \\
\text { at } y=0 \text {,e.g., } t=1 \\
\quad V_{x}, \tau_{x y}, E_{x} \text { and } B_{z} \\
\text { are finite as } \xi \rightarrow-\infty .
\end{gathered}
$$

As a result, the ordinary differential equation (22) with the boundary conditions (23) can be solved precisely by the help of any symbolic software. They sought solutions that would be implemented on a typical model of argon collisionless plasma in the laboratory. 
IV.

\section{Non-Equilibrium Predictions of the Model}

The relation between forces and fluxes can be obtained from a linearity description of non-equilibrium properties. Also, local application of the principle for collisionless plasma needs its state to be about local equilibrium. A finite set of macroscopic variables describes this principle. The irreversible thermodynamics processes for describing the physical phenomena continue to present great significance, such that finding symmetry coefficients of the connection that constitutes Onsager's principle [21, 48]. Starting from H-theorem, the entropy per unit mass $S$ is calculated. It is written bellow in the dimensionless form [27, 34, and 61],

$$
\begin{aligned}
& S=-\int M_{e} \ln M_{e} d \underline{c} \\
& =-\left(\int M_{1} \ln M_{1} d \underline{c}+\int M_{2} \ln M_{2} d \underline{c}\right) . \\
& =-\pi^{\frac{3}{2}}\left[\left(V_{x 1}{ }^{2}+V_{x 2}{ }^{2}\right)-\frac{3}{2}\right]
\end{aligned}
$$

Besides, in the y-direction, the entropy flux component can be acquired as follows,

$$
\begin{aligned}
& J_{y}{ }^{(S)}=-\int \xi_{y} M_{e} \ln M_{e} d \underline{c} \\
& =-\left(\begin{array}{l}
\int \xi_{y} M_{1} \ln M_{1} d \xi+ \\
\int \xi_{y} M_{2} \ln M_{2} d \xi
\end{array}\right) \\
& =\left[\pi\left(V_{x 1}{ }^{2}+V_{x 2}{ }^{2}\right)\right]
\end{aligned}
$$

The entropy production is written using the following law [22, 24],

$$
\sigma=\frac{\partial S}{\partial t}+\vec{\nabla} \bullet \overrightarrow{J^{(S)}} \text {. }
$$

Using the rigid plate Mach number, thermodynamic force as a time-dependent controlling parameter is introduces as in Refs. [27, 62, 64],

$$
\begin{aligned}
& X_{e 11}=\frac{V_{w}}{\sqrt{2 R T}}=\frac{V_{0} \exp \left[-\omega_{1} t\right]}{\sqrt{2 R T}}, \\
& =M a \exp \left[-\omega_{1} t\right]
\end{aligned}
$$

where the relations between the entropy production, forces, and thermodynamic fluxes near the thermodynamic equilibrium has the following form [34, 35, 39],

$$
\sigma=\sum_{l} J_{l}^{(S)} X_{l}, J_{l}^{(S)}=\sum_{j} L_{l j} X_{j} .
$$

Here $L_{l j}$ represent kinetic coefficients, which have to fulfill the Onsager reciprocal relation, coupled with the condition represented by the inequalities $L_{l j}=L_{j l}$ and $L_{l l} \geq 0$ [34]. In the present study, this condition will be satisfied by choosing the coefficient

$$
L 1 \geq 0 \text {. }
$$

In order to investigate the internal energy modification, we will introduce the extended Gibbs formula [27, 31, and 65]. It contained the energy of the electro-magnetic fields as a part of the entire energy balance. What distinguishes the collisionless plasma into paramagnetic and dia-magnetic plasma. Accordingly, if the plasma has unpaired electrons in the molecular orbital system, then the plasma is paramagnetic. Whereas, if all the electrons are paired, the plasma is considered a dia-magnetic. Finally, the internal energy balance, including electro-magnetic field energy, is written in two magnetic states according to the work term of the first law of thermodynamics:

I. Para-magnetic collisionless plasma:

Internal energy modification is expressed in terms of the extensive quantities $P, S$, and $M_{G}$, that represents the thermodynamic coordinates corresponding to the conjugate intensive quantities $E, T$, and $M$, respectively. Using Gibbs formula, the three participants in the internal energy modification are as follows,

$$
d U=d U_{p o l}+d U_{S}+d U_{p a r} .
$$

Here $d U_{p o l}=E d P$ is the internal energy modification due to alteration of polarization, $d P d U_{S}=T d S$ is the internal energy modification due to alteration of the entropy, and $d U_{\text {par }}=P d M$ is the internal energy modification due to the alteration of magnetization, where $M$ is measured from the following differential equation [27,65],

$$
\frac{\partial S}{\partial M_{G}}=-\frac{B}{T} \Rightarrow M_{G}=-\int\left(\frac{T}{B} \frac{\partial S}{\partial y}\right)_{t} d y .
$$

The non-dimensionalized form of Gibbs relation can be obtained by using the dimensionless variables $U^{\prime}=\frac{U}{K T}, M_{G}^{\prime}=M_{G}\left(\frac{1}{e \tau_{e e} V_{T e}}\right), P^{\prime}=P\left(\frac{1}{e \tau_{e e} V_{T e}}\right)$

as follows (after dropping the primes), $d U=d S+f_{1} E d P+f_{1} B d M_{G}$.

II. Dia-magnetic collisionless plasma:

On the other hand, if the collisionless plasma is diamagnetic, the internal energy modification can be investigated. In this case, internal energy representing the thermodynamic coordinates conjugates to the intensive quantities. Therefore, the three participants in the internal energy modification in Gibbs formula are given by,

$d U=d U_{S}+d U_{p o l}+d U_{d i a}$,

here $d U_{d i a}=-M_{G} d B$ is the internal energy modification due to the alteration of the induced magnetic induction and $M_{G}=T \frac{\partial S}{\partial B}[27,65]$.

Thus, the non-dimensional form of $d U$ takes the form, $d U=d S+f_{1} E d P-f_{1} M_{G} d B$,

here $f_{1}=\left(\frac{m_{e} V_{T e}^{2}}{K T}\right), \quad d S=\left(\frac{\partial S}{\partial r}\right) \delta y+\left(\frac{\partial S}{\partial t}\right) \delta t$, and $\delta y=0.1, \delta \quad t=1$.

\section{Discussion}

In the present study, the unsteady manner of rarefied collisionless gaseous plasma is discussed based on the irreversible processes in kinetic theory by applying the exact traveling wave analytical solutions of the VlasovMaxwell equation. Numerical computations are performed according to typical data for collisionless argon plasma [66] as a para-magnetic medium where the argon loses a single electron. It is found that, in a dia-magnetic medium, the argon loses electron pairs. The potential ionizing, in this 
case, is implemented on the argon atoms according to the conditions and parameters bellow:

$T_{0}=1200 \mathrm{~K}, k_{B}=1.380710^{-16} \mathrm{erg} / \mathrm{K}, n_{e}=710^{11} \mathrm{~cm}^{-3}$, and $d=3.8410^{-8} \mathrm{~cm}$ (argon atom diameter). The rest mass and charge of the electron are $m_{e}=9.093 \times 10^{-28} \mathrm{gm}, e=4.810^{-10} e s u$, which used to calculate the dimensionless parameter $\alpha_{0}=9.58 \times 10^{-5}$, the relaxation time is $\tau_{e e}=1.15 \times 10^{-9}$, and the average free path of the collisionless plasma is $\lambda=\frac{1}{\sqrt{2} \pi n_{e} d^{2}}=2.18010^{2} \mathrm{~cm}$, which compared to the electron Debye length $\lambda_{D e}=\sqrt{\frac{K_{B} T_{0}}{4 \pi n_{e} e^{2}}}=2.8510^{-5} \mathrm{~cm}$ $f_{1}=1.66667$. The transformation constants $m=1, l=0.8$, and the rigid plate Mach number $M a=0.05$.

All variables in the present model satisfy the initial and boundary conditions (18), as seen in Figs. (2)-(6), which cannot be exactly examined in the previous study in Ref. [7]. In this reference, authors put restrictions on distance $y$ values and time $t$ that related to each other. That is because of their solutions discontinuity due to the effect of the Heaviside function in the model.

Figure (1) shed light upon the boundary and initial conditions for electrons velocity. As revealed in this figure, at the vicinity of the moving plate, the mean velocity has a value equals to the Mach number of the plane plate $M a=0.05$, which satisfies the conditions of the problem. The velocity, $V_{x}$ gets a severe linear decrement with $y$, while it increases nonlinearly with time $t$. Moreover, the shear stress decreases nonlinearly with time and increases linearly with $y$, as seen in Fig. (2). Figures (3) and (4) demonstrate that the boundary and initial conditions of the induced electrical and magnetic fields are satisfied where $B_{z}(0,0)=E_{x}(0,0)=0$. As shown in Fig. (3), the induced magnetic field is decreasing slightly non-linear with time, $t$ and linearly with distance, $y$. While the induced electric field is increasing linearly with time, $t$ and decreasing linearly with distance, $y$, as seen in Fig. (4).

Investigating the irreversible non-equilibrium thermodynamic properties of the system, we found that: the entropy, $S$ is increasing with time, $t$ that consistent with the Boltzmann H-theorem, the second law of thermodynamics, and Le Chatelier's principle, as shown in Fig. (5). The same consistent satisfy by the entropy production behavior where it is a non-negative value $\sigma \geq 0$, as shown in Fig. (6). As shown in Fig (7), the kinetic coefficient is a non-negative function $L_{11} \geq 0$, which is consistent with the second law of thermodynamics.

Investigating the vital participation in the internal energy alterations of the entire system, we acquired that: all participations satisfies the conditions of the physical problem where $d U_{S}(0,0)=d U_{p o l}(0,0)=d U_{d i a}(0,0)=0$. In the beginning, the system was in equilibrium, as shown in Figs. (8)-(11). It is seen from Fig. (8), that internal energy entropy alteration $d U_{S}$, is smoothly damping with time directly away from the plate when $t \in[0.8,1]$. It has a linear decrement in the vicinity of the moving plate, due to the energy lost to and gained from the electrons and plate, respectively. Fig. (9) shows that the internal energy due to the modification of polarization increases with the time, $t$ and decreases with $y$ to be agreeable for the behavior of the electric fields, see Figs. (4) and (9). While the alteration in internal energy due to the modification of the intensity of the induced magnetic field for the diamagnetic gas $d U_{\text {dia }}$ smoothly varies between two maximum negative values, as illustrated in Fig. (10).. The thermodynamic predictions reveal the order of maximum magnitude ratios

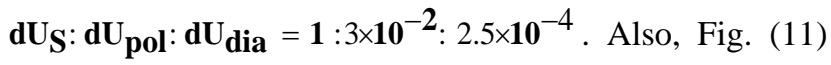
shed light on the behavior of the thermodynamic force.

\section{Conclusions}

The solution of the unsteady Vlasov-Maxwell equations for rarefied collisionless gaseous plasma using the method of moments with the two-sided distribution function together with the Maxwell equations is investigated. That allows calculating the components of the flow velocity and fluxing using the traveling wave exact solution method. The Boltzmann $\mathrm{H}$-theorem is verified using the velocity components into the two-sided distribution functions. The entropy, and entropy production, have been calculated using the underlying thermodynamic principles. Moreover, the ratios between the different internal energy modification participations are evaluated using the Gibbs equations based on the total derivatives of the extensive parameters. Besides, the calculated thermodynamic reveal the order of the prediction of the maximum numerical magnitude ratios between the participants of the internal energy modifications. Therefore, the total derivatives of the extensive values in Gibbs formula are

$d U_{S}: d U_{\text {pol }}: d U_{\text {dia }}=1: 3 \times 10^{-2}: 2.5 \times 10^{-4}$. It is concluded that the internal energies modification effect in $d U_{p o l}$ and $d U_{d i a}$ due to the electro-magnetic fields is small compared with $d U_{S}$. This result is because these self-induced fields are caused by the sudden movement of the rigid plate.

\section{Data Availability}

The data used to support the findings of this study are included within the article.

\section{Conflicts of Interest}

The author declares that there is no conflict of interest regarding the publication of this paper.

\section{Acknowledgments}

This study is supported by the Egyptian Academy of Scientific Research and Technology by the associated grant number (No.6508), under the program of ScienceUP Faculties of Science. 


\section{References}

[1] Taha Zakaraia Abdel Wahid, J. Non-Equilibrium Thermodynamic, 37, 2, (2012), 119-141.

[2] Taha Zakaraia Abdel Wahid and S.K. Elagan, Can. J. Phys. 90: 987-998 (2012).

[3] Taha Zakaraia Abdel Wahid, Canadian Journal of Physics, 91(3):201-210, (2013).

[4] Taha Zakaraia Abdel Wahid " Travelling Waves Solution of the Unsteady Flow Problem of a Rarefied Non-Homogeneous Charged Gas Bounded by an Oscillating Plate." Mathematical Problems in Engineering 2013; (2013). (ID 503729):1-13.

[5] Taha Zakaraia Abdel Wahid "Travelling waves solution of the unsteady problem of binary gas mixture affected by a non-linear thermal radiation field. ", American Journal of Physics and Applications. Vol. 2, No. 6, pp. 121-134, (2014). (DOI: 10.11648/j.ajpa.20140206.13)

[6] Taha Zakaraia Abdel Wahid, "Travelling Waves Solution of the Unsteady Flow Problem of a Collisional Plasma Bounded by a Moving Plate.", Fluid Mechanics, Vol. 4, No. 1, pp. 27-37, (2018). (DOI: 10.11648/j.fm.20180401.14).

[7] Wahid, T.Z.A., El-Malky, F.M., SN Appl. Sci. 2, 791 (2020). https://doi.org/10.1007/s42452-020-2583-9.

[8] Taha Zakaraia Abdel Wahid "Kinetic and Thermodynamic Study of The Thermal Radiation Effect on The Gases." , LAMBERT Academic Publishing, Germany., ISBN: ISBN 978-613-992664-0, (2019).

[9] Taha Zakaraia Abdel Wahid "Kinetic and Thermodynamic Treatments of Unsteady Gaseous Plasma Flows," LAMBERT Academic Publishing, Germany, ISBN: 978-613-9-90736-6, (2018).

[10] Taha Zakaraia Abdel Wahid" Irreversible Thermodynamic of a New Model of the Collision Term of the Boltzmann Kinetic Equation Dealing with Gas Mixture affected by a Centrifugal Field" preprint arXiv:1811.10887, 2018 ( 2018arXiv181110887Z ).

[11] A. H. Khater, A. E. El-Sharif, "Analytical solution of the Rayleigh's flow problem for a highly rarefied gas of a homogeneous system of charged particles" Astrophysics and space 146, 157-162, (1988).

[12] V. M. Zhdanov and V. We. Roldughin. Phys.-Uspekhi, 41, 349, (1998). DOI:10.1070/PU1998v041n04ABEH000383.

[13] P. Bhatnagar, E. Gross, and M. Krook, Phys. Rev. 94, No.3, (1954).

[14] T. Hauser and J. Allen "A DSMC Solver for Rarefied Flow Applications Based On Open FOAM" Open Source CFD International Conference 2008, Berlin, Germany.

[15] B. L. Hass, and D. A. Schmitt, Journal of Spacecraft and Rockets, 31, (6), 980-985, (1994).
[16] D. Rault "Aerodynamics of the Shuttle Orbiter at High Altitudes," Journal of Spacecraft and Rockets, 31, (6), 944-952, (1994)

[17] R. Wilmoth, R. Mitcheltree, and J. Moss, "LowDensity Aerodynamics of the Stardust Sample Return Capsule," AIAA-97-2510, (1997).

[18] J. Moss, R. Blanchard, R. Wilmoth, and R. Braun, "Mars Pathfinder Rarefied Aerodynamics: Computations and Measurements," Journal of Spacecraft and Rockets, 36, (3), 330-339, (1999).

[19] S. Plimpton and T. Bartel "Parallel particle simulation of low-density fluid flow," US Department of Energy Report, No. DE94-007858, (1993).

[20] X. Yang, and J. Yang "Micromachined membrane particle filters," Sensors and Actuators, 184-191, (1999).

[21] A. Frangi, A. Frezzotti, and S. Lorenzana, Computers and Structures 85, 810-817, (2007).

[22] E. Piekos, and K.Breuer "Numerical modeling of micromechanical devices using the direct simulation Monte Carlo method" Journal of Fluids Engineering, 118, 464-469, (1996).

[23] C. Cercignanit, A. Frangi, A. Frezzottit, G. P. Ghiroldil, L. Gibellil, and S. Lorenzana., SENSOR letters, 6, 121-129, (2008).

[24]Alexeenko, S. Chigullapalli, "Implications of Rarefied collisionless gaseous plasma Damping for RF MEMS Reliability", AIP Conference Proc., $1333,27^{\text {th }}$ International Symposium on Rarefied Gas Dynamics, 701-706, (2011).

[25] Taha Zakaraia Abdel Wahid "The Effect of Lorentz and Centrifugal Forces on Gaseous and Plasma." LAMBERT Academic Publishing, Germany, (2017), ISBN: 978-620-2-05504-8.

[26] Taha Zakaraia Abdel Wahid "Kinetic and Irreversible Thermodynamic study of Plasma and Neutral Gaseous.", LAMBERT Academic Publishing, Germany, (2014), ISBN: 978-3-659-62296-0.

[27] A.M. Abourabia and T Z. Abdel Wahid, Can. J. Phys. vol 88, 501-511, (2010).

[28] V.P. Shidlovskiy. Introduction to the dynamics of rarefied gases. Elsevier, NY. 78-85, (1967).

[29] A.G. El-Sakka, R.A. Abdellatif, and S.A. Montasser. Astrophys. Space Sci. 109, 259, (1985). (Doi:10.1007/BF00651272.)

[30] A.H. Khater and A.E. El-Sharif. Astrophys. Space Sci. 146, 157, (1988). (Doi: 10.1007/BF00656989.)

[31] V.V. Aristov. Methods of direct solving the Boltzmann equation and study of non-equilibrium. Kluwer Academic, Dordrecht, Netherlands. (2001).

[32] C. Cercignani. Theory and application of the Boltzmann Equation. Scottish Academic Press, Edinburgh, UK. (1975).

[33] S. Chapman and T.G. Cowling. The mathematical theory of non-uniform gases. Cambridge University Press, Cambridge, UK., (1970).

[34] G. Lebon, D. Jou, and J. Casas-Vazquez. Understanding non-equilibrium thermodynamics: foundations, applications, frontiers. Springer-Verlag, Berlin, Heidelberg, Germany. (2008).

[35] B.C., Eu. Kinetic theory and irreversible thermodynamics. Wiley, New York, USA., (1992). 
[36] A. M. Abourabia, and T. Z. Abdel Wahid, Journal of Non-Equilibrium Thermodynamics, 36, (1), 75-98 (2011).

[37] A. M. Abourabia, and T. Z. Abdel Wahid "Kinetic and Thermodynamic Treatment of a Neutral Binary Gas Mixture Affected by a Nonlinear Thermal Radiation Field." Submitted to the Canadian Journal of Physics, (2011).

[38] A. M. Abourabia, and T. Z. Abdel Wahid, Journal of Non-Equilibrium Thermodynamics, (2011).

[39] V. M. Zhdanov and V. We. Roldughin. Phys.-Uspekhi, 41, 349, (1998). DOI:10.1070/PU1998v041n04ABEH000383.

[40] C. Cercignani. The Boltzmann equation and its application. Springer, New York, USA. (1988).

[41] JA Mclennan. Introduction to non-equilibrium statistical mechanics. Prentice-Hall, Englewood Cliffs, NJ, USA., (1990).

[42] T.G. Cowling. Magnetohydrodynamics. Adam Hilger Ltd., Bristol, UK., (1976).

[43] H. Grad. Commun. Pure Appl. Math. 2, 331, (1949). (Doi:10.1002/cpa.3160020403.)

[44] L. Lees. J. Soc. Ind. Appl. Math. 13, 278, (1965). (Doi:10.1137/0113017.)

[45] L. Lees and C.-Y. Liu. Phys. Fluids, 5, 1137, (1962). (Doi:10.1063/1.1706498.)

[46] E. Wasserstrom, C.H. Su, and R.F. Probstein. Phys. Fluids, 8, 56, (1965). (Doi:10.1063/1.1761102.)

[47] A. H. Khater, A. E. El-Sharif "Analytical solutions of the Rayleigh flow problem for a rarefied gas of a nonhomogeneous system of charged particles" Phy. A: Stat. and Theoretical Physics 150, Issue 3, 592-613, (1988).

[48] Smirnov, B. M., Introduction to plasma physics. Published by Mir Publishers (1977).

[49] K. Nishikawa and M. Wakatani," Plasma physics," Springer- Verlag. (2000).

[50] B. Chakraborty" Principles of plasma mechanics," Wiley Eastern Ltd., New Delhi, India. (1978).

[51] B. M. Smirnov," Plasma Process and Plasma Kinetics.", WILEY-VCH Verlag GmbH \& Co. KGaA, Weinheim (2007).

[52] P. Bhatnagar, E. Gross, and M. Krook. Phys. Rev. 94, 511, (1954). (Doi:10.1103/PhysRev.94.511.)

[69] A.G. Sitenko, Electro-magnetic fluctuation in plasma, Academic Press, New York. (1967).
[53] J.D. Huba. NRL plasma formulary. Naval Research Laboratory, Washington, DC, (2019).

[54] S.We. Braginski "Transport processes in a plasma "Reviews of Plasma Physics, New York, pp. 205-255, (1965).

[55] We. H. Hutchinson "Principles of Plasma Diagnostics "Cambridge University Press, (2009).

[56] T.J.M. Boyd, J.J. Sanderson "The Physics of Plasmas" Cambridge Uni. Press, New York, (2003).

[57] Zhdanov, V. M. and Roldugin, V. We., The method of moments and the non-equilibrium thermodynamics of rarefied gaseous, J. Exp. Theor. Phys., 86(6) 11411148 (1998).

[58] E.M. Purcell. Electricity and magnetism. 3rd ed. McGraw-Hill Book Co., Singapore, (1965).

[59] J. Gratton, S. M. Mahajan, and F. Minotti "NonNewtonian Gravity Creeping Flow" International Centre for Theoretical Physics, Trieste (Italy), 1-17, (1988).

[60] A. M. Abourabia, A. M. Morad, Exact traveling wave solutions of the van der Waals normal form for fluidized granular matter. Physica A 437: 333-350 (2015).

[61] G. Nugroho, A. M. S. Ali, and Z. A. Abdul Karim, World Acad. of Sci., Eng. And Tec. (2009).

[62] A.M. Abourabia, M.A. Mahmoud, and W.S. Abdel Kareem. J. Appl. Math. 2, 141, (2002). (Doi:10.1155/ S1110757X02108023.)

[63] F.M. Sharipov. Physica A, 203, 457, (1994). (Doi:10.1016/0378-4371(94)90010-8.)

[64] V. V. Vysotsky and V. We. Roldughin," Mechanodiffusion in a Slightly Rarefied Gas Mixture," Physica A 209, 25-41, (1994).

[65] P. Van der Linde. Periodica Polytechnica Ser. Chem. Eng., 12, 97, (1998).

[66] S. Yonemura and K. Nanbu. Jpn. J. Physiol. 40, 7052, (2001). (Doi:10.1143/JJAP.40.7052.)

[67] V. S. Galkin "Application of The Chapman-Enskog Method to The Case of a Binary Two-Temperature Gas Mixture" Izv. AN SSSR. Mekhanika Zhidkosti I Gaza, 2, 6, 58-63, (1967).

[68] CO. Bennett and J.E. Myers, Moment, Heat and Mass Transfer, 3rd ed. McGraw-Hill Book Co., Singapore. (1988). 

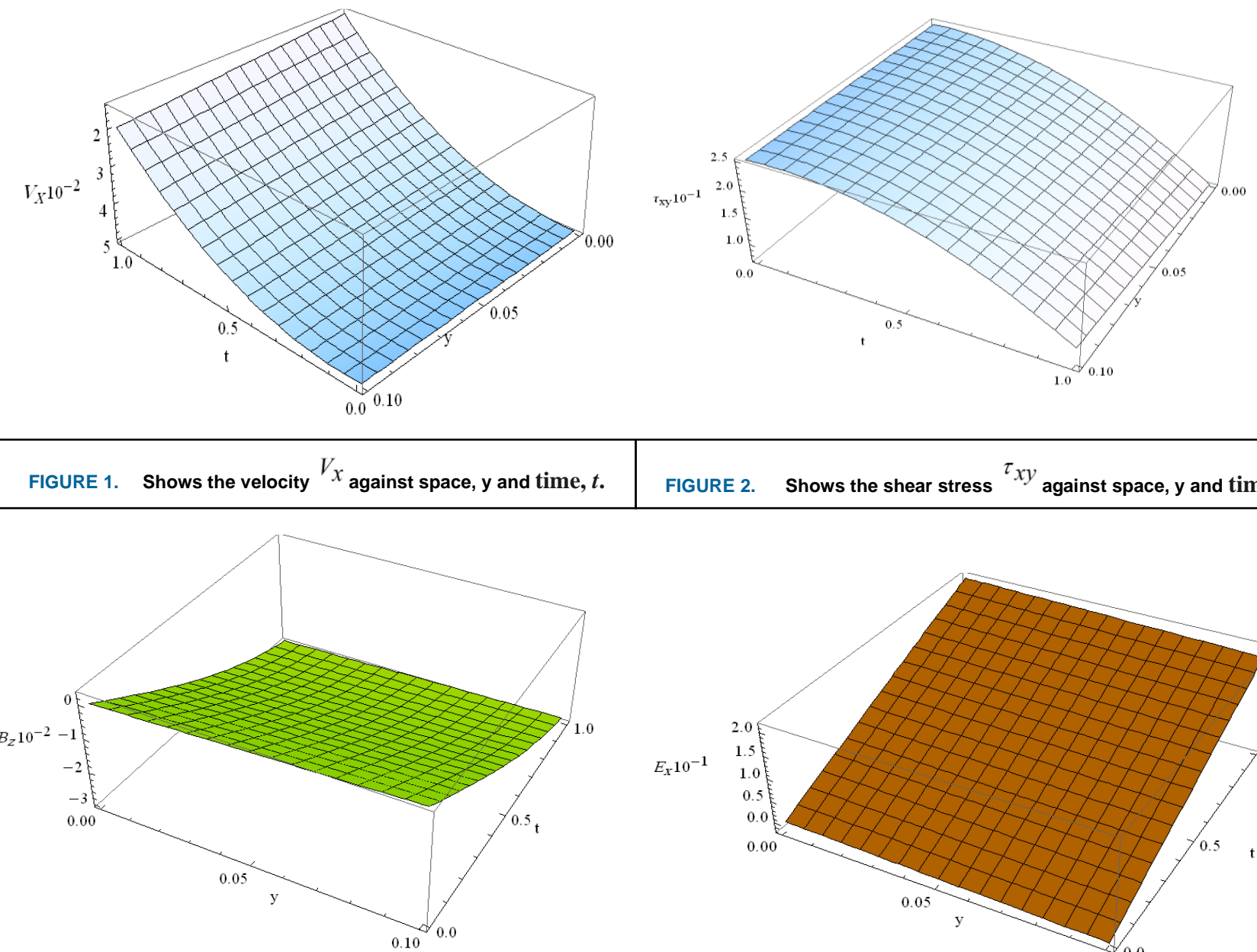

FIGURE 2. Shows the shear stress ${ }^{\tau}{ }_{x y}$ against space, $\mathbf{y}$ and time,

FIGURE 3. Shows the induced magnetic field $\boldsymbol{B}_{z}$ against $\mathrm{y}$ and $\mathrm{t}$.

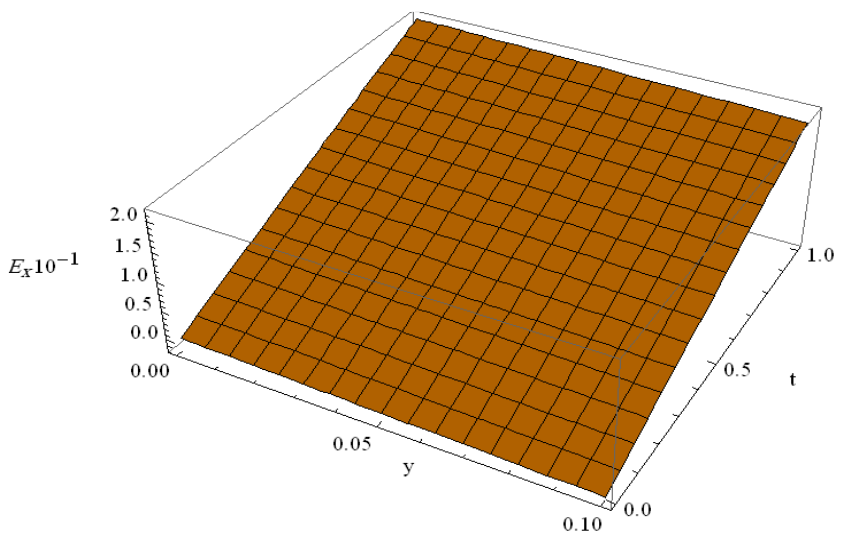

FIGURE 4. Shows the induced electric field $E_{x}$ against $\mathbf{y}$ and $\mathbf{t}$.

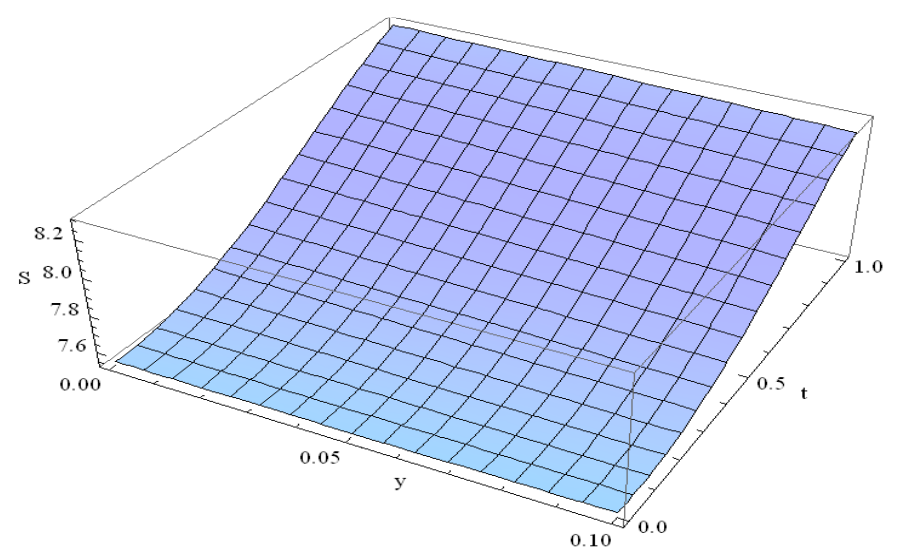

FIGURE 4. Shows the entropy, $\mathrm{S}$ against space, $\mathrm{y}$ and time, $\mathrm{t}$. 

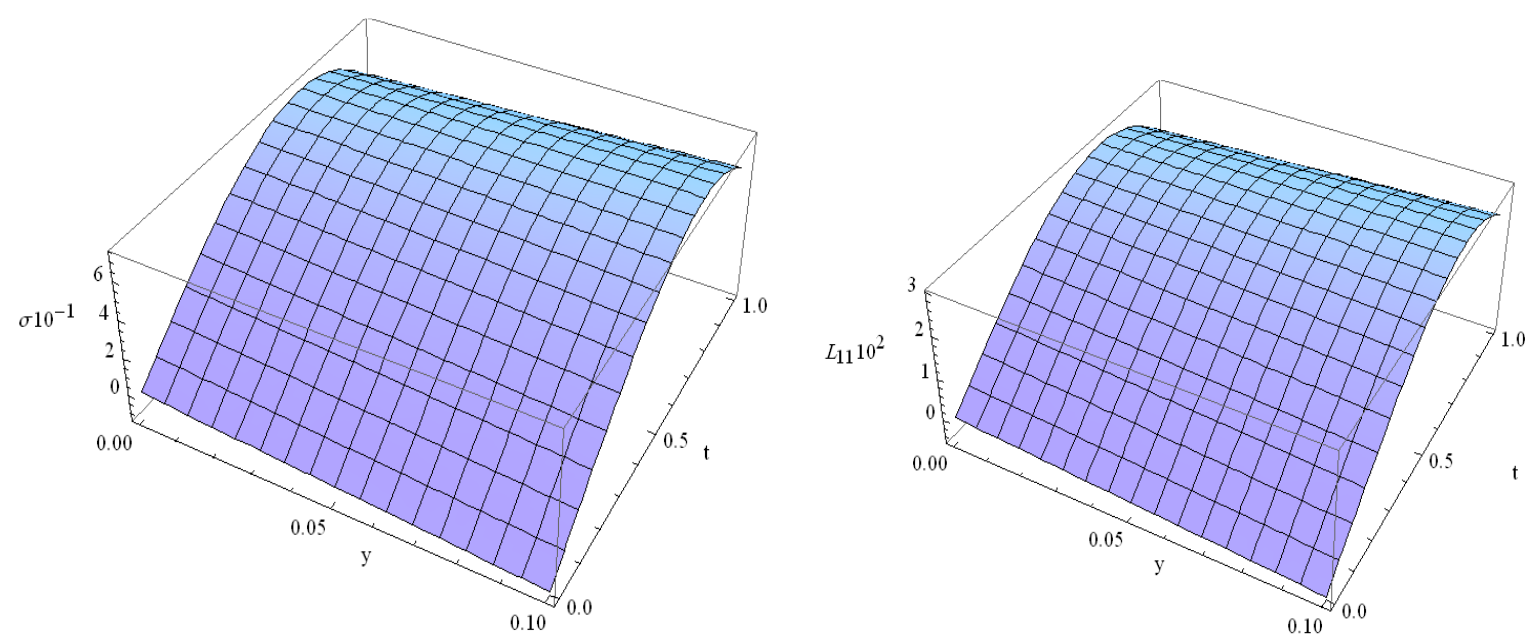

FIGURE 5. Shows the entropy production, $\sigma$ against space, $y$ and time, $t$.

FIGURE 6. Shows the kinetic coefficient, $L_{11}$ against space, $\mathrm{y}$ and time, $\mathrm{t}$.
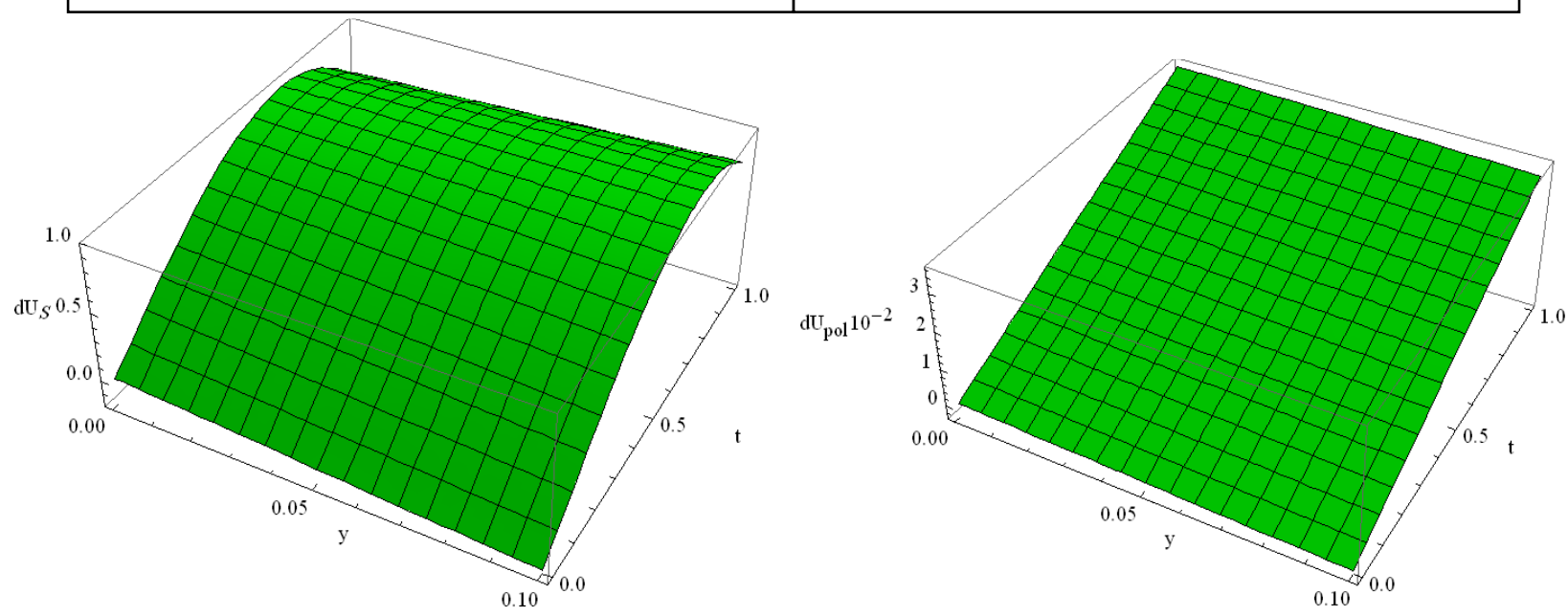

FIGURE 7. Shows the internal energy change $d U_{s}$ against space, $y$ and time, $t$.

FIGURE 8. Shows the internal energy change $d U_{p o l}$ against space, $y$ and time, $t$.
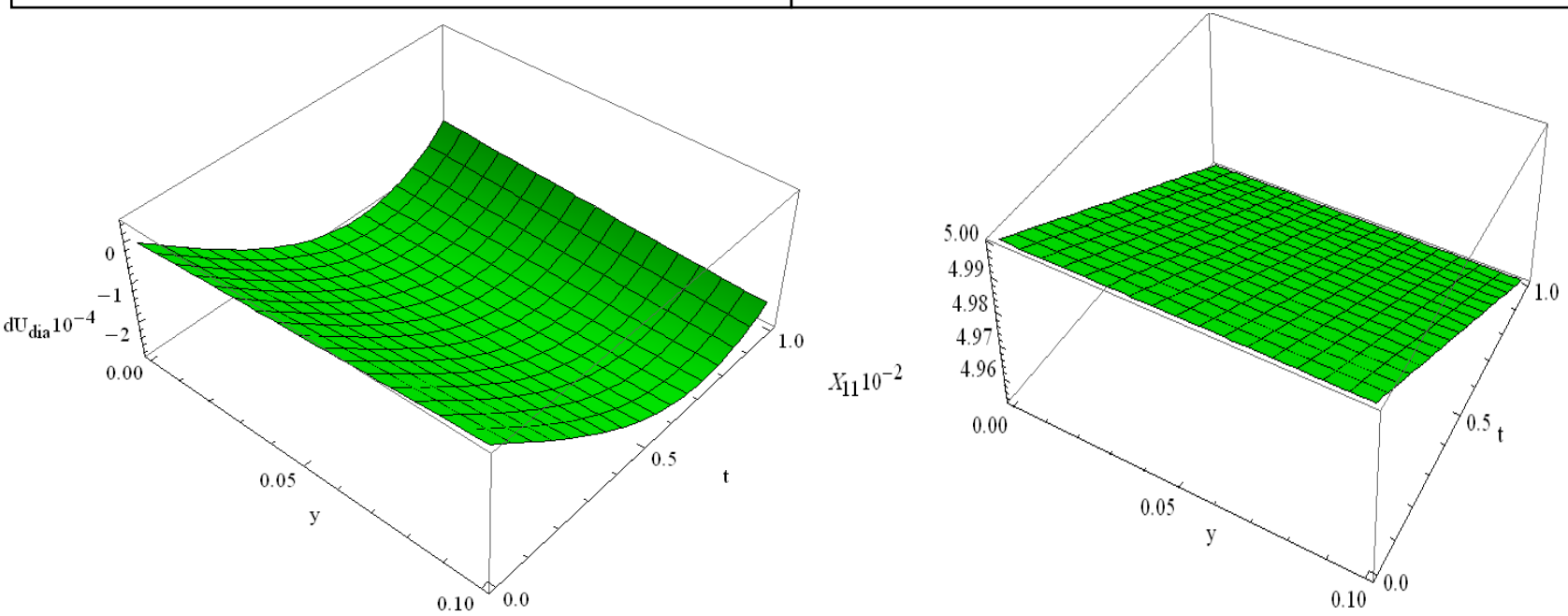

FIGURE 9. Shows the internal energy change $d U_{\text {dia }}$ against space, $y$ and time, $t$.

FIGURE 10. Shows thermodynamic force $X_{11}$ against space, $y$ and time, t. 